\title{
Autologous arteriovenous fistula creation for haemodialysis in small children
}

\author{
Dragan Piljic $^{1 *}$ Nail Sehic ${ }^{1}$, Dilista Piljic ${ }^{1}$, Fahrudin Sabanovic ${ }^{2}$, Mate Petricevic ${ }^{3}$ and Gordan Samoukovic ${ }^{4}$ \\ ${ }^{1}$ University Clinical Center Tuzla, Tuzla, Bosnia and Herzegovina \\ ${ }^{2}$ University Hospital Center Zenica, Zenica, Bosnia and Herzegovina \\ ${ }^{3}$ University Hospital Center Zagreb, Zagreb, Croatia \\ ${ }^{4}$ McGill University, Montreal, Canada
}

\begin{abstract}
A 11-years old female with growth problem and chronic renal failure was admitted for arteriovenous fistula (AVF) formation for long-term haemodialysis in the pediatric ward. During her hospital stay, continue to have a cuffed central venous catheter (CVC) as the first choice for vascular access.

AVF the cubital artery - vena cephalica was performed with a standard end-to-side anastomosis with a continuous running 8 -0 prolene monofilament suture.

The patient was discharged on the second post operative day, subsequent to a normal AVF primary patency.

We recommend that vascular and pediatric Hemodialysis centers establish a strategy supporting AVF creation in small children when peritoneal dialysis is contraindicated and pre-emptive kidney transplantation is not feasible.
\end{abstract}

\section{Introduction}

Chronic renal failure is a condition in which kidney function is irreversibly damaged. Patients do not recover because the nephron's function has been irreversibly progressing for years, even when the original disease that damaged the kidneys is no longer active [1].

Kidney disease can affect children in various ways, ranging from treatable disorders without long-term consequences to life-threatening conditions. Acute kidney disease develops suddenly, lasts a short time, and can be serious with long-lasting consequences or may go away completely once the underlying cause has been treated. Chronic kidney disease (CKD) does not go away with treatment and tends to get worse over time. CKD eventually leads to kidney failure, described as endstage renal disease (ESRD) when treated with a kidney transplant or blood-filtering treatments called dialysis [2].

CKD is one of the most severe chronic diseases in a child. The prevalence in the population aged 0 to 18 is $50-80$ children per million. It is estimated that the incidence of progressive kidney disease leading to terminal (end-stage) renal failure is 6-8 children per million pediatric population under 18 years of age [1].

Kidney disease usually gets worse slowly, and symptoms may not appear until kidneys are badly damaged. In the late stages of kidney disease, as the condition is near kidney failure, these symptoms may appear: Itching, Muscle cramps, Nausea and vomiting, Not feeling hungry, Polydipsia, Pale skin, Loss of appetite, Swelling in feet and ankles, Polyuria or oliguria, Trouble sleeping and concentrating, Weight loss, GI bleeding, Hypertension, Convulsion [3].

From birth to age 4, birth defects and hereditary diseases are the leading causes of kidney failure. Between ages 5 and 14, kidney failure is most commonly caused by hereditary diseases, nephrotic syndrome, and systemic diseases. Between ages 15 and 19, diseases that affect the glomeruli are the leading cause of kidney failure, and hereditary diseases become less common [2].

The diagnosis is based on laboratory evidence of renal dysfunction, which is sometimes followed by a kidney biopsy. Treatment is primarily focused on the underlying disease, but includes water and electrolyte control, and often dialysis and / or transplantation [4].

Children with kidney disease that leads to kidney failure must receive treatment to replace the work the kidneys do. The two types of treatment are dialysis and transplantation [2].

Dialysis is the process of filtering wastes and extra fluid from the body. Sometimes, a transplanted kidney may stop working, and the child may need to return to dialysis. Hemodialysis and peritoneal dialysis are the two types of dialysis. Hemodialysis helps control blood pressure and helps the body maintain the proper balance of important minerals, such as potassium, sodium, calcium, and bicarbonate [2].

For hemodialysis, a surgeon creates an access to the bloodstream, called a vascular access - AVF, several weeks or months before the first treatment [2].

${ }^{*}$ Correspondence to: Dragan Piljic, M.D., Ph.D., Department of Cardiovascular Surgery, University Clinical Center Tuzla, Bosnia \& Herzegovina, Tel: +38735303202; E-mail: dragan.piljic@dr.com

Key words: arteriovenous fistula, chronic kidney disease, hemodialysis

Received: November 13, 2020; Accepted: December 22, 2020; Published: December 25, 2020 


\section{Case report}

A 11-years old female with growth problem and chronic renal failure was admitted for AVF formation for long-term haemodialysis in the pediatric ward (Figure 1). She was weight $27 \mathrm{~kg}$ and height 126.7 $\mathrm{cm}$ (Figure 1). Her blood pressure was $140 / 100 \mathrm{mmHg}$, with heart rate $100 / \mathrm{min}$. Five years earlier, she had noticed sinus tachicardia and swelling mass on front of the neck.

Ultrasound showed enlarging right lobe Thyroid gland measuring $11.2 \times 10.7 \times 38.7 \mathrm{~cm}$ in diameter and left lobe measuring $10.1 \times 8.8 \times 36.9$ $\mathrm{cm}$. Volume thyroid gland was $3.9 \mathrm{ml}$.

During her hospital stay, continue to have a cuffed central venous catheter (CVC) as the first choice for vascular access.

AVF the cubital artery - vena cephalica was performed with a standard end-to-side anastomosis with a continuous running 8-0 prolene monofilament suture (Figure 2). Local anesthesia was used for the procedure. To decrease the vasospastic response from arterial clamping and vessel dissection, tourniquet occlusion was used for inflow control.

The patient was discharged on the second post operative day, subsequent to a normal AVF primary patency.

Child is evaluated approximately 5 weeks after AVF formation to assess the maturation of the fistula by ultrasound examination.

The six-month postoperative period AVF was primary patency.

\section{Discussion}

Patients with chronic renal failure require permanent vascular access in the form of direct or indirect arteriovenous fistula. The best vascular approach is AVF. It is a surgically established connection between an artery and a vein (most often the radial artery - cephalic vein) with the aim of obtaining greater blood flow in the superficial veins. In doing so, the veins dilate due to exposure to higher arterial

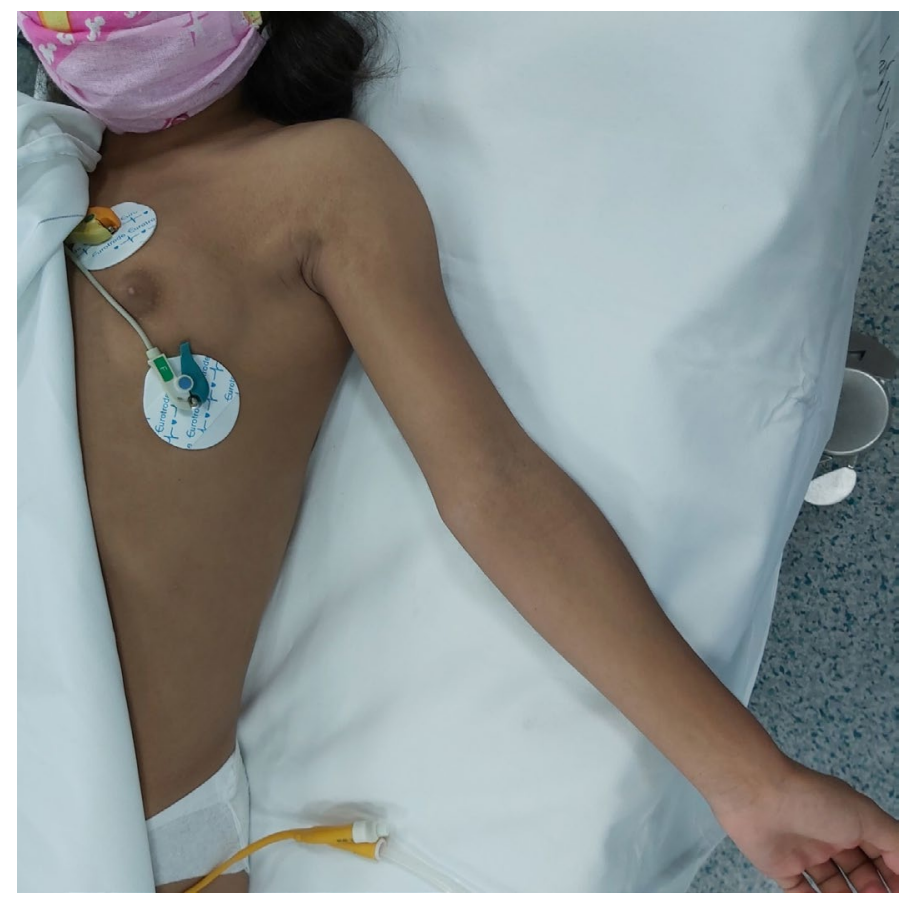

Figure 1. A 11-years old female with growth problem and chronic renal failure

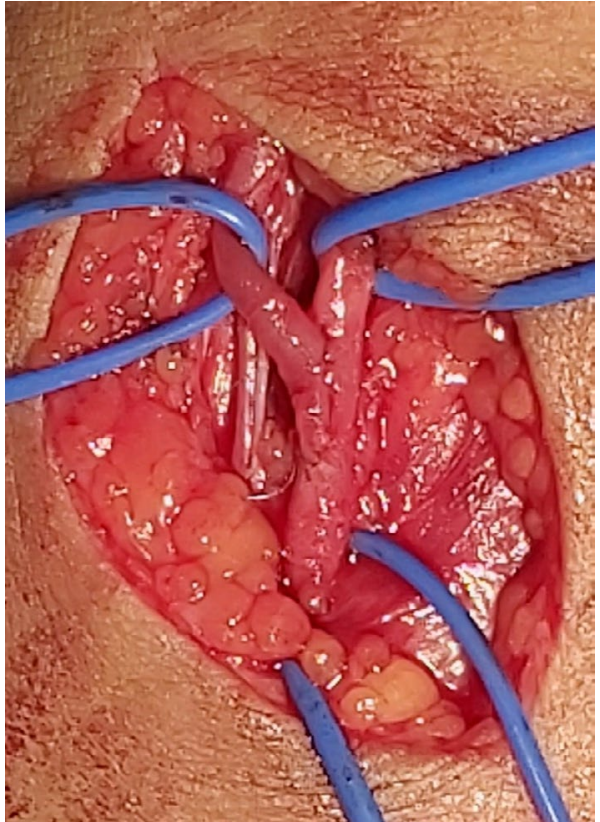

Figure 2. Completed microsurgical anastomosis the brachial artery - basilic vein in small child

pressure, making them more readily available. AVF allows the flow of large volumes of blood through the hemodialysis device (more than $300 \mathrm{ml} / \mathrm{min}$ ) and achieving the required dose of dialysis. During hemodialysis, the veins are punctured with two needles, one of which is used to take and the other to return blood to the patient. Dilation and change in the structure of the veins, the so-called. "AVF maturation" takes several weeks, and sometimes several months, before the AVF is ready for use. Catheters placed in large veins, most often the jugular vein, less often the subclavian or femoral vein, are used for emergency access to blood vessels. A central venous catheter is a good short-term solution for patients who need hemodialysis and do not have AVF ready for use. The patient can check the flow through the vascular access once to twice a day. When you touch a spot on the skin beneath which the vascular approach is located, a pulsating flow or noise of flow can be palpated. The flow through the vascular access can be checked by palpation or a stethoscope, which can be used to hear the blood flow [5].

To ensure longevity and good flow through a permanent vascular approach, the following should be considered:

1. Blood pressure must not be measured on the arm (s) on which a permanent vascular access has been created,

2. Tightly fitted clothing on the arm or leg with permanent vascular access should be avoided and should not sleep in a position that allows direct pressure on the vascular access [6].

When receiving hemodialysis treatments, a child may have problems with the vascular access (AVF), such as

- infection

- poor blood flow

- blockage from a blood clot

- small vessels

- spasm and arterial dissection

- aneurysmosis 
These problems can keep treatments from working, and the child may need to undergo more than one surgery for the access to work properly [2].

\section{Radial-to-cephalic arteriovenous fistulas}

The incision is longitudinal, half way between the cephalic vein and the radial artery in the wrist. The nerves must be carefully preserved. The vein is freed; a ligature being placed on the collateral branches avoiding electric coagulation that could damage the vascular trunk itself. Saline irrigation must be frequent in order to prevent drying, and vessels are only handled by the adventitia. After section of the vein above a ligature, a longitudinal posterior incision of approximately $10 \mathrm{~mm}$ in length is made in the proximal vein. The anterior wall of the artery is exposed. A longitudinal arteriotomy is made with a disposable ophthalmologic scalpel and completed with scissors. If a few drops of blood appear they are rinsed with heparin saline and dried with small sponges. The anastomosis is constructed with four running sutures, by suturing first the proximal angle. The running suture is left loose at the beginning so as to leave the lumen open. Frequent use of the zoom facilitates this very precise part of the operation and the needle must be repositioned in the needle holder for each passage through a vessel. Both proximal running sutures (posterior and anterior) are stopped at the middle of the arteriotomy. The anastomosis is then completed, starting from the distal angle. The tourniquet is then released. If there is a leak, an additional suture is added. Patency is confirmed by Doppler ultrasound, as the murmur can be absent during the first postoperative hours, particularly in small children. It is very important to ascertain that the vein is not stenosed at the upper limit of the dissection [6].

\section{Variations}

One running suture anastomosis: The proximal suture is performed first, starting at the middle of the posterior wall of the arteriotomy, proceeding to the proximal angle, and down to the middle of the anterior wall. The distal suture is then completed. This technique gives excellent vision of the arterial lumen.

It may be necessary to surgically transpose the vein subcutaneously 2 months later in small children with thick subcutaneous tissue.

\section{Ulnar-to-basilic arteriovenous fistulas}

A sufficient length of the vein must be freed, as it is situated at a distance from the artery. It can be helpful to place the forearm in flexion at the elbow during dissection. The artery is approached by partial excision of the flexor carpi ulnaris. The interval before puncture is longer than with a radial-to-cephalic AVF.

\section{Brachial-to-cephalic arteriovenous fistulas}

A transverse incision at the anterior aspect of the elbow exposes the medial branch of the cephalic vein, which must be sufficiently freed to allow it to come into proximity with the brachial artery without traction. Here again, using a pneumatic tourniquet avoids having to free the artery.

\section{Variations}

Anastomosis between the brachial artery and the cephalic vein by means of the medial branch of the basilic vein is preferable. This will be perfused backwards in the absence of valves. Both vessels, which follow almost superimposed routes, are approached by a short antecubital incision. The vein is divided as high as possible and, after minimal dissection, its lower segment is anastomosed to the brachial artery. It is essential to ligate the perforating antecubital vein to avoid the risk of high flow with cardiac overload. Ligation of the upper part of the forearm cephalic vein is also necessary to avoid the risk of retrograde perfusion of the forearm, which would impair venous drainage of the hand. Both these risks mean that retrograde AVF and side to side AVF at the elbow must not be used.

\section{Brachial-to-basilic arteriovenous fistulas with subcutaneous transposition}

The basilic vein follows a route which rapidly becomes deep above the elbow, meaning that it is often undamaged. Its use for angioaccess necessitates surgical transposition, even in the thinnest children. Its transposition is much easier when it is performed during a second stage, the first consisting of a simple AVF at the elbow which results in dilatation of the vein and thickening of its walls. The initial AVF is constructed using the medial branch of the basilic vein at the elbow or, if necessary, on its lateral branch. Transposition performed 2 months later requires a longitudinal incision on the medial side of the upper arm. Collaterals of the veins are ligated and divided. The vein is tunnelled very superficially along a straight line. Reimplantation on the artery is performed as low as possible. An interval of 3 weeks must be left before puncturing.

\section{Femoral-to femoral arteriovenous fistulas, with transposition}

This type of vascular access is rarely used. Superficial transposition of the femoral vein is usually in a straight line with an anastomosis on the distal superficial femoral artery.

\section{Arteriovenous bridge-graft}

In this case a graft (biograft or Polytetrafluoroethylene-PTFE) is implanted between an artery and a vein. It is located under the skin in such a way as to be accessible to puncture [7].

\section{Conclusion}

The construction of an arteriovenous fistula is the method of choice for hemodialysis treatment in patients with chronic renal failure. AVF is feasible in small children, it provides long-lasting and good access and good blood flow for hemodialysis treatments improves the quality of life, which is extremely important in childhood. We recommend that vascular and pediatric Hemodialysis centers establish a strategy supporting AVF creation in small children when peritoneal dialysis is contraindicated and pre-emptive kidney transplantation is not feasible.

\section{Informed consent}

The patient provided written informed consent for publication of the figures.

\section{Conflicts of interest}

The authors declare that there is no conflict of interest

\section{Funding}

This work received no specific grant from any funding agency in the public, commercial, or not-fot-profit sectors.

\section{References}

1. www.svjetskidanbubrega.org

2. www.NIDDK.NIH.gov 
3. www.kidneyfund.org

4. www.msd-prirucnici.placebo.hr

5. www.zzjzpgz.hr
6. Frank W, Logerfo, Robert C Davis (1979) Radial-Cephalic Arteriovenous Fistula Technique. Arch Surg 114: 346

7. Bourguelot P (2006) Vascular access in children: The importance of microsurgery for creation of autologous arteriovenous fistulae. European Journal of Vascular and Endovascular Surgery 32: 696-700.

Copyright: (C2020 Piljic D. This is an open-access article distributed under the terms of the Creative Commons Attribution License, which permits unrestricted use, distribution, and reproduction in any medium, provided the original author and source are credited. 